\title{
Acesso aos serviços de saúde: abordagens, conceitos, políticas e modelo de análise
}

\author{
Access to health services: \\ approaches, concepts, policies and analysis model
}

Marluce Maria Araújo Assis ${ }^{1}$

Washington Luiz Abreu de Jesus ${ }^{2}$

\footnotetext{
${ }^{1}$ Departamento de Saúde, Universidade Estadual de Feira de Santana. Av. Transnordestina s/n, Novo Horizonte. 44036-900 Feira de Santana BA. marluce.assis@ pesquisador.cnpq.br ${ }^{2}$ Faculdade de Medicina da Bahia, Universidade Federal da Bahia.
}

\begin{abstract}
Access to health services is a multifaceted and multidimensional issue involving political, economic, social, organizational, technical and symbolic aspects in establishing access to universal healthcare. This theoretical review paper intends to discuss the different approaches, analyze the context and policies for special groups on access, marking an analysis model delineated by the above aspects, from readings on the topic in question. This analysis reveals a diversity of approaches to access the formulation and implementation of public policies and their potential for changing the organization of the health system. We identified progress in reducing inequalities in health and increased access to the network of the Unified Health System (SUS), especially in primary care. There were also limitations related to accessibility, fragmentation, decentralization and regionalization of the service network, with inadequacies in the process of care and attention to specific groups, and regional disparities. Finally, the analysis model proposed seeks to develop a critical stance to reflect and intervene in health practices and services, with the objective goal being responsible, comprehensive, effective, equitable and quality healthcare.
\end{abstract}

Key words Health care, Access to health services, Unified Health System
Resumo $O$ acesso aos serviços de saúde é um tema multifacetado e multidimensional envolvendo aspectos politicos, econômicos, sociais, organizativos, técnicos e simbólicos, no estabelecimento de caminhos para a universalização da sua atenção. Pretendemos discutir, nesta revisão teórica, as diferentes abordagens, a análise do contexto e as políticas voltadas para grupos especiais sobre acesso, demarcando um modelo de análise pautado nos aspectos acima referidos, a partir releituras sobre a temática em questão. As análises revelam uma diversidade de abordagens sobre acesso na formulação e implementação de políticas públicas e no seu potencial para mudanças na organização do sistema de saúde. Identificaram-se avanços na diminuição das iniquidades e na ampliação do acesso na rede do Sistema Único de Saúde (SUS), em especial na atenção básica. Observaram-se também limites relacionados a acessibilidade, fragmentação, descentralização e regionalização da rede de assistência, com inadequação no processo de acolhimento e da atenção a grupos específicos, além das assimetrias regionais. O modelo de análise proposto busca desenvolver uma postura crítica para refletir e intervir nas práticas e serviços, tendo como imagem objetivo uma atenção responsável, integral, resolutiva, equânime e de qualidade.

Palavras-chave Sistema de saúde, Acesso aos serviços de saúde, Sistema Único de Saúde 


\section{Introdução}

O acesso aos serviços de saúde tem sido objeto de análise na literatura internacional ${ }^{1,2}$, principalmente no contexto de crise econômica que se instala na última década, demarcando a existência de barreiras aos usuários como filas para marcação de consulta e atendimento, bem como estratégias para sua superação. Observa-se $o$ acesso aberto ou avançado em relação à organização da marcação de consultas para o mesmo dia em que o usuário procura o atendimento, buscando intervir para a redução do agendamento em longo prazo e diminuir o tempo de espera para a consulta médica. Este sistema procura equilibrar a oferta em relação à demanda, adequando às práticas desenvolvidas na Atenção Primária à Saúde (APS), além de planos de contingência para circunstâncias incomuns que são apresentadas no cotidiano dos serviços de saúde.

No contexto brasileiro, a temática em discussão mostra que é mais um exemplo de que a legalidade de uma proposta não assegura a sua implementação: "não se cria igualdade por Lei, ainda que não se consolide a igualdade sem a Lei”3. Apesar da Carta Magna Brasileira de 1988 assegurar a saúde enquanto direito universal a ser garantido pelo Estado, a despeito dos avanços conquistados, ainda se convive com a realidade desigual e excludente do acesso ao Sistema Único de Saúde (SUS). A garantia na legislação brasileira foi apenas mais uma etapa alcançada na construção do SUS, para se concretizar o direito à saúde é necessário ter como alicerce um modelo social fundamentado na "solidariedade humana e na igualdade social"3.

Na prática ainda existe um acesso "seletivo, focalizado e excludente" ${ }^{\text {"4 }}$. No entanto, estudos ${ }^{5-7}$ evidenciam as oportunidades de uso de serviços de saúde antes e após a implementação do SUS e apresentam avanços e limites na garantia do seu acesso universal ${ }^{5,7-17}$. Os limites associados, principalmente, a fatores socioeconômicos ou pelas barreiras geográficas e os avanços relacionados a ampliação da oferta de serviços na rede básica de saúde. Porquanto, ainda, persistem significativas diferenças entre regiões e municípios brasileiros.

$\mathrm{O}$ que se quer chamar a atenção aqui, diz respeito a sua relação com as condições de vida da população, nutrição, habitação, poder aquisitivo e educação, englobando a acessibilidade aos serviços, que extrapola a dimensão geográfica, abrangendo também o aspecto econômico, relativo aos gastos do usuário com o serviço, o aspecto cultural envolvendo normas e técnicas ade- quadas aos hábitos da população e o aspecto funcional pela oferta de serviços adequados às necessidades da população ${ }^{18}$. Nesse sentido, engloba uma compreensão multidimensional ${ }^{19}$, com caráteres social e político, necessariamente presentes nas formulações e execução de políticas de saúde como a disponibilidade de serviços, acessibilidade, organização do serviço, acolhimento, necessidades e aceitação da população, buscando sempre a resolubilidade da atenção em todos os níveis de complexidade do sistema.

As dimensões de análise por serem múltiplas e de caráter sócio, econômico e cultural, configuram-no como norteador na construção de políticas públicas. "As dimensões de análise da categoria acesso em busca de uma totalidade concreta, teriam que ser alicerçadas no princípio da equidade, no estabelecimento de caminhos para a universalização da atenção, regionalização, hierarquização e participação popular"20.

O presente texto busca discutir as diferentes abordagens, a análise do contexto e as políticas voltadas para grupos especiais sobre acesso aos serviços de saúde, apresentando um modelo de análise (Figura 1), organizado em 05 (cinco) dimensões: política, econômico-social, técnica, organizacional e simbólica, a partir de releituras teóricas sobre a temática em questão.

\section{Acesso aos serviços de saúde e a diversidade de abordagens}

As diferentes abordagens de análise sobre acesso aos serviços de saúde demonstram o nível de pluralidade e complexidade do tema, considerando que: "acesso é um conceito complexo, muitas vezes empregado de forma imprecisa, e pouco claro na sua relação com o uso de serviços de saúde"21.

Para categorizar o acesso e analisar as condições de acessibilidade, Giovanella e Fleury ${ }^{22}$ adotam como abordagem teórica quatro dimensões explicativas (política, econômica, técnica e simbólica), na tentativa de articular o referencial teórico e a definição conceitual a ser utilizada, culminando em quatro modelos teóricos: a dimensão econômica - modelo economicista - é referente à relação entre oferta e demanda; dimensão técnica - modelo sanitarista-planificador relativo à planificação e organização da rede de serviços; dimensão política - modelo sanitarista-politicista - relativo ao desenvolvimento da consciência sanitária e da organização popular; e a dimensão simbólica - modelo das representações sociais acerca da atenção e ao sistema de 


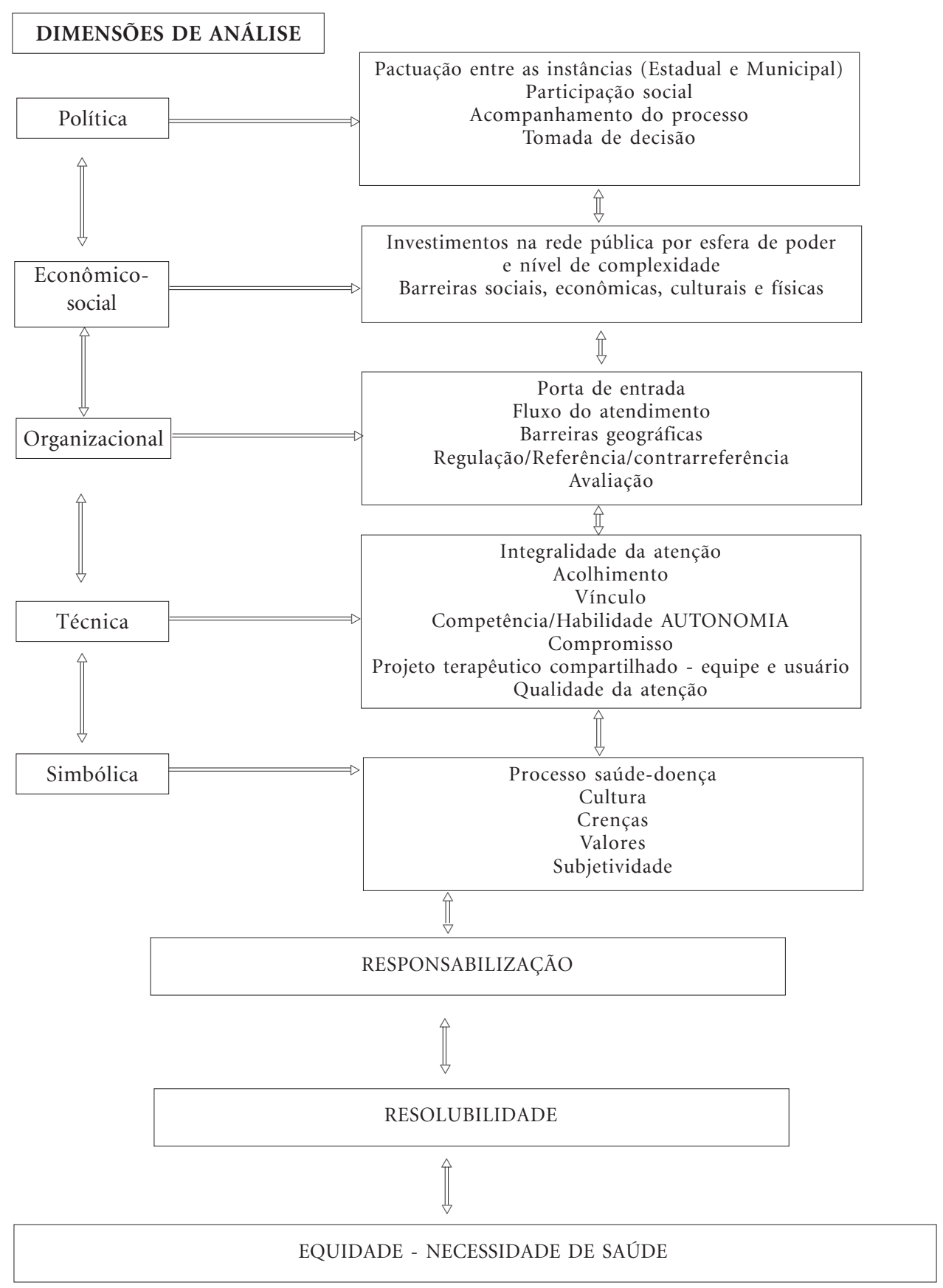

Figura 1. Modelo de análise: acesso universal aos serviços de saúde.

saúde. Referem-se, também, às dimensões específicas do acesso como: disponibilidade, acessibilidade, adequação funcional, capacidade financeira e aceitabilidade.

A disponibilidade é percebida como relação entre o volume e o tipo de serviços existentes, o volume de usuários e o tipo de necessidade. A acessibilidade assemelha-se à descrita por Unglert ${ }^{18}$, caracterizada pela relação entre localização da oferta e dos usuários, distância entre eles, forma de deslocamento e custos. Adequação funcional é "entendida como a relação entre o modo 
como a oferta está organizada para aceitar os usuários e a capacidade/habilidade destes em acomodarem-se a esses fatores e perceberem a conveniência dos mesmos" ${ }^{20}$, associando-se aos fatores capacitantes, tendo como recorte de análise a capacidade financeira dos serviços e a relação entre os custos e a sua oferta. E, finalmente, a aceitabilidade, entendida como a relação entre as atitudes dos usuários, trabalhadores de saúde e práticas destes serviços.

No entanto, para Unglert ${ }^{18}$, o acesso geográfico é caracterizado pelo tempo de deslocamento e distância entre a residência do usuário e o serviço de saúde, seguindo o conceito de acessibilidade geográfica e de territorialização. O acesso econômico é caracterizado a partir de facilidades e dificuldades que o usuário encontra para obter $\mathrm{o}$ atendimento como forma e custos de deslocamento, de procedimentos e obtenção de medicamentos e condição social atual. A dimensão técnica envolve desde a entrada propriamente dita aos serviços de que o cidadão necessita, incluindo-se os horários previstos de atendimento e a qualidade da atenção, reinterpretados por Ramos e $\operatorname{Lima}^{23}$ que enfatiza o papel facilitador do acolhimento.

Hortale et al. ${ }^{24}$ admitem a hipótese de que se o sistema de saúde é descentralizado vai permitir maior acesso dos usuários ao sistema e operacionaliza a categoria acesso, abrangendo os vários planos de produção dos serviços (subsistema de decisão, de operação/produção de serviços e subsistema de informação e retroalimentação), associando com as diferentes instâncias dos serviços (central/local; população/usuários). As dimensões que compõem a categoria acesso abarcam fatores bem distintos da mera acessibilidade organizacional aos serviços, como: a participação popular e o controle social, a equidade, a coerência dos serviços com as necessidades da população, as estratégias, as táticas e a alocação de recursos e a autonomia.

As diferentes abordagens sobre acesso sugerem um quadro consolidado embasado na literatura, propondo que o acesso represente o "grau de ajuste" entre os serviços de saúde e a comunidade; "acesso é a liberdade de usar serviços de saúde" e é representado por três dimensões: disponibilidade, acessibilidade e aceitabilidade ${ }^{19}$. A disponibilidade caracteriza-se por todos os fatores de um serviço específico ao alcance do usuário. A acessibilidade refere-se aos custos diretos e indiretos dos cuidados em relação à capacidade de pagamento do usuário; e, a aceitabilidade do serviço que abrange o subjetivo, o social e o cultural, tais como o grau que um determinado ser- viço é culturalmente seguro, por isso, defendem que a informação é essencial para que um potencial acesso transforme-se em uso de serviços.

Já na definição do uso de serviços de saúde, e suas formas de utilização, o acesso é mediado por três fatores individuais: fatores predisponentes, capacitantes e de necessidades de saúde. Os fatores predisponentes são aqueles que existem previamente ao surgimento do problema de saúde e afetam a predisposição das pessoas para usar serviços de saúde como variáveis sociodemográficas (idade, gênero, raça, hábitos, entre outros). Os fatores capacitantes são condicionados pela renda, cobertura securitária pública ou privada, e pela oferta de serviços, ou seja, o meio disponível para as pessoas usarem os serviços. Os fatores determinantes referem-se às necessidades de saúde que podem ser explicadas pelas condições diagnosticadas por profissionais ou pela autopercepção ${ }^{25}$. Nessa análise, o uso de serviços de saúde é uma expressão positiva do acesso, no entanto, a utilização dos serviços depende também dos fatores individuais.

No que se refere à $\mathrm{APS}^{26}$, o acesso pode ser relacionado com as diversas possibilidades de adentrar aos serviços de saúde, as quais estariam implicados com a localização da unidade de saúde, a disponibilidade de horários e os dias em que a unidade atende, bem como a possibilidade de atendimento a consultas não-agendadas e a percepção que a população tem em relação a estes aspectos do acesso (se são adequados ou não).

Se considerar a possibilidade do atendimento à saúde ser dividida em fases, o acesso aos serviços seria considerado a primeira fase a ser superada pelo usuário, na busca de atendimento para resolução de problemas, ou a satisfação de uma necessidade dentro do sistema de saúde, envolvendo algumas questões, tais como: disponibilidade ou não do serviço, a distância, e quais práticas estão sendo ofertadas para o atendimento dos usuários; quais são os recursos disponíveis, qual a capacitação técnica dos profissionais que atuam na unidade de saúde e de que forma têm se responsabilizado pelos problemas da população, a quem e como devem prestar serviço ${ }^{26}$.

Em relação à oferta e à demanda dos serviços de saúde, muitas vezes, são utilizados na perspectiva "economicista" de uma relação prestadorusuário, sendo o acesso um elemento transformador da realidade e, não apenas, como possibilidade de consecução do cuidado. Estes conceitos se ampliam e extrapolam a dimensão econômica, abrangendo aspectos de ordem política, social e cultural. 
As demandas por serviços de saúde resultam da conjugação de fatores sociais, individuais e culturais prevalentes na população ${ }^{27}$. Em uma perspectiva discursiva, a demanda é uma mescla de direitos, necessidades e desejos, que assumem conotações distintas de acordo com o processo específico de interanimação dialógica ${ }^{28}$. A oferta de serviços de saúde está relacionada à disponibilidade, ao tipo e à quantidade de serviços e recursos destinados à atenção ${ }^{29}$.

Há estudos ${ }^{30-32}$ que abordam este desequilíbrio entre demanda e oferta nos diversos níveis de complexidade da rede assistencial. Apesar de a ampliação da rede básica ter contribuído para melhor acessibilidade geográfica, verificou-se desproporção entre oferta, capacidade de atendimento e demanda ${ }^{33}$. Desta forma, mantém-se o modelo clássico de assistência a doenças em suas demandas espontâneas, devido à limitação da assistência integral, face a ausência de uma rede regionalizada de referência e contrarreferência ${ }^{33}$.

Essa diversidade de abordagem reflete a importância da categoria acesso na formulação e implementação de políticas públicas e o seu potencial para mudanças na organização do SUS. Nesse sentido, identificaram-se como fatores limitantes desta dimensão: baixa acessibilidade, sistema fragmentado, focalizado, ausência de hierarquia, descentralização e regionalização da rede de assistência, ausência de equidade, de acolhimento e permeando estes diversos fatores, a excludente capacidade financeira.

\section{Acesso aos serviços de saúde: análise de contexto}

Na conjuntura atual, a Estratégia Saúde da Família (ESF), é constituída como o primeiro nível de atenção às pessoas com o encargo de "reestruturar a atenção prestada pelo SUS em todo o território nacional e como estratégia de implantação do SUS em localidades onde não avançou a política de saú$\mathrm{de}^{3{ }^{34}}$. Estudos ${ }^{35-37}$ mostram que a sua implantação tem contribuído para a diminuição das iniquidades em saúde e ampliação do acesso à APS.

No entanto, observam-se alguns limites do acesso aos serviços básicos de saúde: a baixa capacidade de interação entre as equipes da atenção básica (unidades tradicionais e ESF); a desintegração entre a ESF e os demais níveis de complexidade do sistema; e a pouca competência da APS em suas relações com outros setores da sociedade e do governo ${ }^{35}$.

Além das dificuldades de acesso apresentadas devido à oferta inadequada, existem aquelas devi- do à presença sistemática da demanda reprimi$\mathrm{da}^{30,38}$. "No Brasil, estudos têm revelado que pessoas inseridas nos grupos de renda mais baixa procuram menos os serviços de saúde ou apresentam menor probabilidade de usá-los"39. Por meio de pesquisa que utiliza metodologia baseada na epidemiologia ${ }^{27}$ pode-se avaliar estas demandas reprimidas ou prever novas demandas: "os serviços de saúde deverão se preparar para o atendimento especializado da população idosa, pois é no grupo de idosos de baixa renda que se concentram as pessoas de alta morbidade e de graves limitações de atividades físicas”. Entretanto, avaliar desigualdades no uso dos serviços básicos de saúde implica, sobretudo, extrapolar os estudos de demanda para alcançar também os indivíduos fora do sistema, identificar possíveis focos de exclusão e compensar as diferenças existentes ${ }^{40}$.

Em uma conjuntura específica, da região Nordeste do Brasil, o modo como são produzidas as ações de saúde e a maneira como os serviços se organizam para produzi-las e distribuí-las são resultantes das diversas políticas de confronto de interesses. Sendo a população múltipla em suas demandas, em uma rede insuficiente e subutilizada, muitas dificuldades se interpõem, no dia-adia, entre a disponibilidade planejada e a utilização efetiva dos serviços de saúde, para pacientes portadores de hanseníase que necessitam de atenção especial e não tem uma atenção integral e resolutiva nos serviços desta realidade estudada ${ }^{41}$.

Em pesquisa ${ }^{29}$ realizada sobre a oferta e demanda dos serviços de saúde bucal na ESF de um município da Região Nordeste da Bahia, observa-se a incerteza no acesso e a desconfiança na qualidade dos serviços, afastando segmentos sociais do sistema público, que acabam optando pelo desembolso direto ou por um plano de saúde privado. Pois que, "a oferta é insuficiente para $o$ atendimento de uma demanda que sempre teve dificuldade de acesso aos serviços de saúde bucal, mantendo, portanto, muitos destes usuários excluídos do sistema, até mesmo porque, para aumentar a oferta, é preciso pelo menos, uma equipe de saúde bucal para cada uma de saúde da família”29.

Esses fatos tornam-se mais preocupantes quando analisados conjuntamente com o estu$\mathrm{do}^{42}$ que avalia as transferências de recursos públicos para os planos e seguros privados de saúde. "A maior parte da oferta de serviços hospitalares é de propriedade privada: $62 \%$ dos estabelecimentos com internação e $68 \%$ dos leitos do país. Segundo a Assistência Médico Sanitária (MAS) e o Instituto Brasileiro de Geografia e Es- 
tatística (IBGE), no Brasil, 69\% dos estabelecimentos privados que contam com internação e $65 \%$ dos leitos privados são contratados pelo SUS. Entretanto, esses estabelecimentos também são contratados pelos planos e seguros privados de saúde. Os dados acima retratam, portanto, que no sistema de cobertura duplicada existente no país, o segmento suplementar disponibiliza uma oferta de serviços hospitalares e, principalmente, de equipamentos de alta e média complexidade muito maior do que a observada no SUS". O referido trabalho demonstra que para este nível de atenção o acesso torna-se mais dependente da capacidade financeira do usuário, e, portanto, mais seletivo e excludente.

Mesmo considerando que grande parte de unidades ambulatoriais brasileiras são de propriedade estatal ${ }^{42}$, a distribuição destes serviços ainda não ocorre de maneira uniforme entre os diferentes Estados brasileiros. A desigualdade no uso dos serviços de saúde entre as duas regiões geográficas deve-se à maior dificuldade de acesso e/ou menor oferta de serviços na Região Nordeste ${ }^{43}$.

Trabalhos ${ }^{31,44}$ que analisaram o efeito da oferta nas internações hospitalares, a chamada demanda induzida pela oferta, concluíram que o efeito desta pode induzir ou reprimir a demanda hospitalar. Assim, quando a oferta for insuficiente, internações justificadas por necessidades de saúde podem deixar de ocorrer; da mesma forma, internações desnecessárias podem estar acontecendo. Tais aspectos acentuam as iniquidades em todo o país, segundo levantamento no Sistema de Informação Ambulatorial do SUS (SIA/SUS): “enquanto na atenção básica o Sudeste tem uma produção 1,5 vezes maior que a do Nordeste (semelhante à diferença da população destas regiões), na média complexidade esta diferença é 2,2 vezes e na alta complexidade, de 3,1 vezes" 38 .

$\mathrm{Na}$ atenção hospitalar ainda se mantém diferenças de acesso segundo grupo de renda, sinalizando que houve aumento da desigualdade social na utilização destes serviços no Brasil entre 1998 e $2003^{45}$. "Grupos que apresentam maiores necessidades de saúde são justamente aqueles que têm maior dificuldade de acessar e utilizar os serviços de saúde" ${ }^{46}$. E, quando os acessam, percebe-se que os indivíduos mais pobres tendem a procurá-los mais por problemas de doença, e não por motivo de prevenção ou exames de rotina, como as pessoas de maior poder aquisitivo ${ }^{47}$.

A possibilidade de usar uma determinada assistência é associada à renda do indivíduo, assim como à cobertura por plano de saúde, o que reproduz as desigualdades sociais no interior do sistema, na medida em que as chances de escolha no consumo de serviços estão limitadas por estes fatores $^{7}$. É o paradoxo do sistema de saúde brasileiro, ao tempo em que, o Estado brasileiro idealiza e formaliza um sistema universal de saúde, transfere recursos para o setor privado, principalmente na média e alta complexidade, que se especializa cada vez mais nas áreas de alto custo, transformando o sistema num serviço segmentado, fragmentado, configurado a partir de múltiplos pagadores ${ }^{42,48,49}$.

\section{Acesso aos serviços e às políticas voltadas para grupos específicos}

Discutir acesso relacionado a políticas voltadas para grupos específicos é procurar apreender a concepção de equidade. Equidade em saúde é compreendida como diretriz de organizar ações e serviços de saúde distintos e direcionados para grupos populacionais desiguais socialmente, com forte traço de "discriminação positiva" para superar estas desigualdades injustas através da formulação e implementação de determinadas ações ${ }^{50}$.

A proposição de políticas de saúde para grupos específicos gera polêmica por ser, a princípio, contrária à universalidade defendida na Carta Magna. "Verifica-se que importantes justificativas teóricas são impostas para a adequação dos serviços às demandas, porém o acesso à APS não deve lançar mão de nenhum método ou processo que eleja populações prioritárias, visto que, no sistema, esse nível é considerado a porta de entrada" 37 .

Sorj $^{51}$, contrariando tal expectativa, faz um alerta decisivo no balizamento de ações inclusivas: "a demanda de direitos realizada pelas novas identidades coletivas, quando associada a reivindicações distributivas, apresenta risco de despolitização e da erosão do espaço público, pois essas identidades questionam a política como produção de uma utopia comum e transformam o sistema de representação em um processo de agregação de interesses de fragmentos sociais".

Contudo, para alguns dos grupos de risco a própria evolução agressiva da patologia, ou o grande potencial de contágio, exige condições de acesso aos serviços de saúde diferenciados, como é o caso de pessoas portadoras de Doenças Sexualmente Transmissíveis ${ }^{52}$, infecção por vírus HIV/ aids $^{50,53}$ e Hanseníase $^{54}$ que necessitam de diagnóstico e intervenção precoce. Outras patologias, mesmo crônicas como a Diabetes Mellitus e a Hipertensão Arterial Sistêmica, necessitam de diferenciação positiva devido às graves consequências 
produzidas se não forem controladas. A utilização de serviços de saúde pelos portadores de problemas crônicos, em sua maior parte idosos, é consideravelmente maior do que a observada entre a população em geral, especialmente nas internações hospitalares, situação que não se reflete apenas no uso de serviços odontológicos por idosos e no acesso aos serviços de saúde dos que vivem em áreas rurais ${ }^{55-59}$. Observa-se a necessidade de atendimento domiciliar, considerando as barreiras físicas e de dependências de outras pessoas para se deslocar para a unidade de saúde, entre os portadores de doenças crônicas e idosos ${ }^{55}$.

As dificuldades de acesso a serviços de saúde para diagnóstico de Tuberculose (TB) em municípios do Brasil foram analisadas nas regiões Nordeste e Sudeste, evidenciando as desigualdades geográficas na utilização de serviços de saúde, cuja avaliação ocorreu em dois momentos, antes da criação do SUS e depois da sua implantação, a partir de 1996. Observaram-se redução das desigualdades geográficas no uso de serviços de saúde nas duas regiões, a região Sudeste apresentou desempenho superior à região Nordeste. Além disso, a descentralização das ações de TB para o Programa Saúde da Família não apresenta desempenho satisfatório para o acesso ao diagnóstico, bem como a forma de organização da atenção não foi fator determinante para garantia de acesso ao diagnóstico precoce da $\mathrm{TB}^{60}$.

A população penitenciária é uma das que apresenta situações de iniquidades de acesso mais críticas. "Nesse ambiente onde a circulação de detentos é restrita e os profissionais de saúde evitam circular [...] a desvalorização do preso e de sua queixa é acompanhada de uma avaliação informal da demanda de assistência por parte dos agentes de segurança penitenciária, o que acaba por resultar em uma modulação do acesso ao serviço baseada em critérios estranhos à saúde"61.

A questão de gênero também coloca como um diferencial ao acesso de serviços de saúde em muitos estudos de demanda, privilegiando a população feminina. Entre as causas estão os valores subjetivos como a incompatibilidade da demonstração de fraqueza, medo com a masculinidade; o papel histórico de que homem não pode adoecer porque tem que sustentar a família; e a falta de identificação masculina com os serviços de saúde devido às ações preventivas se dirigir quase que exclusivamente para mulheres, os horários de atendimento não beneficiarem os trabalhadores e utilizarem as farmácias ou prontos-socorros porque esses responderiam mais objetivamente às suas demandas ${ }^{62-64}$. Para além das barreiras comportamentais, as pessoas portadoras de deficiência ou com mobilidade reduzida merecem cuidado diferencial do sistema de saúde para o desenvolvimento de ambientes acessíveis que vão além das barreiras arquitetônicas, pois dependem também da atitude inclusiva ${ }^{65}$.

É um grande desafio para o sistema de saúde a efetivação de um acesso equitativo, cada segmento social tem diferentes demandas produzidas por processos sociais de exclusão, nem sempre percebidas pelo poder público. Quando estas demandas são percebidas, muitas vezes a gestão não tem acúmulo reflexivo, para formular políticas.

Para grupos sociais organizados as dificuldades não são menos díspares, pois, "segundo a percepção das famílias assentadas e acampadas, o SUS não tem atendido as necessidades de saúde da maioria delas, principalmente pela dificuldade do acesso aos serviços. Para esse grupo, o atendimento de suas necessidades se dá após reivindicações e pressões sobre os governos." ${ }^{66}$. A despeito de sozinho não conseguir extinguir as desigualdades sociais, a construção concreta de um SUS com acesso universal e equitativo pode ser uma ferramenta política e social privilegiada para este combate.

Chama a atenção, a quantidade de produções sobre acesso a serviços odontológicos ${ }^{11,17} \mathrm{e}$ acesso para a população idosa ${ }^{55,56}$. Isto aponta para uma preocupação, por parte dos autores, acerca da oferta insuficiente de serviços de saúde bucal no SUS, em especial na ESF; e sobre a população idosa que, historicamente, representa uma demanda reprimida e que só tende ao aumento das necessidades de saúde, com o envelhecimento populacional. Discutem-se muitos números, índices, teorias, mas questões acerca da sociedade desigual e injusta na qual o SUS está inserido e que determina o seu acesso excludente, seletivo e focalizado, não são objetos de análise.

Destaca-se o papel dos usuários, dos trabalhadores de saúde e gestores como os protagonistas na efetivação do acesso aos serviços de saúde; e do controle social que deve apontar e buscar soluções para as demandas invisíveis e para os principais pontos de exclusão ao acesso que a população enfrenta neste árduo caminho em busca de solução para suas demandas.

As políticas de saúde devem ser capazes de eliminar a barreira financeira entre os serviços e a comunidade, de enfrentar a mercantilização do setor saúde e a desproporção oferta/demanda existente; e construir uma rede de atenção regionalizada e hierarquizada que garanta o acesso universal, equitativo e integral. 
As desigualdades de acesso encontram-se como um dos principais problemas a serem enfrentados para que o SUS funcione efetivamente, conforme os princípios e as diretrizes estabelecidos. Apesar da realidade cruel do acesso aos serviços de saúde, de acordo com necessidades socialmente determinadas, é possível construir a consciência cidadã, derrotar o conformismo social, por meio da emancipação de sujeitos históricos capazes de intervir nesta realidade, e finalmente, garantir o acesso universal e equitativo como construção social no atendimento às necessidades da população.

\section{Considerações finais}

A discussão teórica apresentada possibilitou desenhar um modelo de análise (Figura 1), que toma como dimensões as questões políticas, econômico-social, técnicas, organizativas e simbólicas. Com estas, busca-se compreender as políticas formuladas e sua execução, envolvendo a gestão com tomada de decisão e participação da sociedade, além das práticas operadas no dia a dia dos serviços de saúde: como os serviços se organizam, como os trabalhadores atuam e as subjetividades que transversalizam estas relações, com seus componentes simbólicos e representacionais no atendimento às demandas e necessidades dos usuários SUS.

A dimensão política busca evidenciar o comprometimento dos formuladores de políticas com o acesso universal aos serviços de saúde, a pactuação entre as instâncias de poder, envolvendo governo, sociedade civil, prestadores de serviços públicos e/ou privados e instituições formadoras de recursos humanos em saúde, através do acompanhamento do processo e da tomada de decisão partilhada entre os diferentes sujeitos com representação política com transparência no processo decisório. Estas ações estratégicas podem promover a superação de carências históricas da oferta nos serviços de saúde, em todos os níveis de complexidade do sistema de saúde.

No tocante à dimensão econômico-social, esta pode ser analisada levando em consideração os investimentos na rede pública por nível de complexidade (básico, médio e alta complexidade), com definição das responsabilidades de financiamento por esfera de poder (federal, estadual e municipal). Não obstante, possibilitar interações objetivas, traduzidas na referência interestadual e intermunicipal da oferta e da demanda concretas aos serviços de saúde. Nesse sentido, é preci- so a identificação das barreiras econômicas, sociais, culturais e físicas para proporcionar equidade na dinâmica da organização dos serviços em seus diferentes níveis de complexidade do sistema. Nessa perspectiva, o processo de avaliação não pode prescindir da participação ativa e democrática dos sujeitos que produzem e consomem os serviços de saúde.

A dimensão relacionada à organização da rede de saúde sustenta-se na capacidade dos serviços de saúde em dar respostas objetivas aos problemas demandados pelos usuários. Para tanto, é necessário fortalecer a "porta de entrada” no nível básico da atenção, demarcando os fluxos dos atendimentos organizados a partir das demandas epidemiológica, sanitária e social. A regulação teria que responder de fato a estas, com garantia do atendimento nos diferentes níveis de complexidade. Além disso, é preciso facilitar o acesso dentro dos limites geográficos de cada território social, integrando serviços e práticas por meio da referência e contrarreferência na rede SUS.

A dimensão técnica objetiva responsabilizar os trabalhadores de saúde, os gestores de saúde, os prestadores públicos e/ou privados, situando o usuário do SUS no centro do processo da atenção à saúde. Para esta mudança busca-se a apreensão de dispositivos da integralidade da atenção como: acolhimento, vínculo e autonomia da equipe e usuário, indicando um projeto terapêutico compartilhado. O acolhimento é definido "como uma possibilidade de construir uma nova prática em saúde, compreendendo-o como ações comunicacionais, atos de receber e ouvir a população que procura os serviços de saúde, dando respostas adequadas a cada demanda em todo o percurso da busca; desde a recepção e o atendimento individual ou coletivo, até o encaminhamento externo, o retorno, a remarcação e a alta" ${ }^{67}$. O vínculo $^{68}$ é compreendido como "uma ferramenta que agencia as trocas de saberes entre o técnico e o popular, o científico e o empírico, o objetivo, o subjetivo, convergindo-os para a realização de atos terapêuticos conformados a partir das sutilezas de cada coletivo e de cada indivíduo, favorecendo outros sentidos para a integralidade da atenção à saúde". A autonomia é constituída pela "substituição do prazer do objeto pelo prazer da representação no processo de constituição de sujeitos humanos e autônomos, com capacidade de se autoperceber e perceber o outro em sua dimensão individual e coletiva, atravessada pela criatividade e realização de solidariedade" ${ }^{69}$.

A quinta e última dimensão de análise do acesso, a simbólica, adentra no campo da subjetivi- 
dade em sua dinâmica relacional, envolvendo a compreensão do processo saúde-doença, cultu$\mathrm{ra}$, crenças e valores de sujeitos e grupos sociais que vivem em diferentes territórios nas áreas onde os serviços se organizam para ofertar a atenção loco-regionais.

Essas dimensões congregam para o atendimento das necessidades de saúde, tendo como imagem objetivo uma atenção responsável, integral, resolutiva, equânime e de qualidade.

\section{Colaboradores}

Participaram da elaboração deste artigo MM Assis, delineando o tema e construindo o texto, sua fundamentação e desenvolvimento; WLA Jesus, na revisão sistemática e identificação do referencial bibliográfico, construção e ajuste da versão final.

\section{Agradecimento}

Artigo é parte da Pesquisa financiada pelo CNPq, intitulada: Acesso com qualidade aos serviços de saúde na Estratégia Saúde da Família em dois cenários da Bahia, sob a coordenação da primeira autora.

\section{Referências}

1. Kopach R, De Laurentis P-C, Lawley M, Muthuraman K, Ozsen L, Rardin R, Wan H, Intrevado P, Qu $\mathrm{X}$, Willis D. Effects of clinical characteristics on successful open access scheduling. Health Care Manag Sci 2007; 10(2):111-124.

2. Knight AW, Padgett J, George B, Datoo MR. Reduced waiting times for GP: two exemples of "advanced access" in Australia. Med J Aust 2005; 183(2):101-103.

3. Fleury S. Saúde e democracia: a luta do CEBES. São Paulo: Lemos Editorial; 1997.

4. Assis MMA, Villa TCS, Nascimento, MAA. Acesso aos serviços de saúde: uma possibilidade a ser construída na prática. Cien Saude Colet 2003; 8(3):815823.

5. Mendoza-Sassi R, Béria JU. Utilización de los servicios de salud: una revisión sistemática sobre los factores relacionados. Cad Saude Publica 2001; 17(4): 819-832.

6. Abreu DMX, César CC, França EB. Relação entre as causas de morte evitáveis por atenção à saúde e a implementação do Sistema Único de Saúde no Brasil. Rev Panam Salud Pública 2007; 21(5):282-291.

7. Travassos C, Viacava F, Fernandes C, Almeida CM. Desigualdades geográficas e sociais na utilização de serviços de saúde no Brasil. Cien Saude Colet 2000; 5(1):133-149.

8. Lima MADS. Acesso e acolhimento em unidades de saúde na visão dos usuários. Acta Paul Enferm 2007; 20(1):12-17.

9. Santos AM, Assis, MMA, Rodrigues, AAO, Nascimento MAA, Jorge MSB. Linhas de tensões no processo de acolhimento das equipes de saúde bucal do Programa Saúde da Família: o caso de Alagoinhas, Bahia, Brasil. Cad Saude Publica 2007; 23(1):75-85. 
10. Marsiglia RMG, Silveira C, Carneiro Junior N. Políticas sociais: desigualdade, universalidade e focalização na saúde no Brasil. Saúde Sociedade 2005; 14(2):69-76.

11. Barros AJD, Bertoldi AD. Desigualdades na utilização e no acesso a serviços odontológicos: uma avaliação em nível nacional. Cien Saude Colet 2002; 7(4):709-717.

12. Kassouf AL. Acesso aos serviços de saúde nas áreas urbana e rural do Brasil. Rev Econ Sociol Rural 2005; 43(1):29-44.

13. Manhães ALD, Costa AJL. Acesso a e utilização de serviços odontológicos no Estado do Rio de Janeiro, Brasil, em 1998: um estudo exploratório a partir da Pesquisa Nacional por Amostra de Domicílios. Cad Saude Publica 2008; 24(1):207-218.

14. Ribeiro MCSA. Perfil sociodemográfico e padrão de utilização de serviços de saúde para usuários e não-usuários do SUS - PNAD 2003. Cien Saude Colet 2006; 11(4):1011-1022.

15. Travassos C, Oliveira EXG, Viacava F. Desigualdades geográficas e sociais no acesso aos serviços de saúde no Brasil: 1998 e 2003. Cien Saude Colet 2006; 11(4):975-986.

16. Fontanella F, Speck FP, Piovezan NA, Kulkamp IC. Conhecimento, acesso e aceitação das práticas integrativas e complementares em saúde por uma comunidade usuária do Sistema Único de Saúde na cidade de Tubarão/SC. ACM Arq Catarin Med 2007; 36(2):123-136.

17. Leal RB, Tomita NE. Assistência odontológica e universalização: percepção de gestores municipais. Cien Saude Colet 2006; 11(1):155-160.

18. Unglert CVS. O enfoque da acessibilidade no planejamento da localização e dimensão de serviços de saúde. Rev Saude Publica 1990; 24(6):445-452.

19. Thiede M, McIntyre D. Information, communication and equitable access to health care: a conceptual note. Cad Saude Publica 2008; 24(5):1168-1173.

20. Abreu de Jesus W L, Assis MMA. Revisão sistemática sobre o conceito de acesso nos serviços de saúde: contribuições do planejamento. Cien Saude Colet 2010; 15(1):161-170.

21. Travassos C, Martins M. Uma revisão sobre os conceitos de acesso e utilização de serviços de saúde. Cad Saude Publica 2004; 20(Supl. 2):S190-S198.

22. Giovanella L, Fleury S. Universalidade da Atenção à Saúde: acesso como categoria de análise. In: Eibenschutz C, organizadora. Política de Saúde: o público e o privado. Rio de Janeiro: Fiocruz; 1995. p. 177-198.

23. Ramos DD, Lima MADS. Acesso e acolhimento aos usuários em uma unidade de saúde de Porto Alegre, Rio Grande do Sul, Brasil. Cad Saude Publica 2003; 19(1):27-34.

24. Hortale VA, Pedroza M, Rosa MLG. Operacionalizando as categorias acesso e descentralização na análise de sistemas de saúde. Cad Saude Publica 2000; 16(1):231-239.
25. Andersen RM, Newman JF. Societal and individual determinants of medical care utilization in the United States. Milbank Mem Fund Q 1973; 51(1):95-124.

26. Starfield B. Atenção Primária. Equilíbrio entre necessidades de saúde, serviços e tecnologia. 2a Edição. Brasília: Ministério da Saúde; 2004.

27. Sawyer DO, Leite IC, Alexandrino R. Perfis de utilização de serviços de saúde no Brasil. Cien Saude Colet 2002; 7(4):757-776.

28. Spink MJP. Sobre a possibilidade de conciliação do ideal da integralidade nos Cuidados à Saúde e a cacofonia da demanda. Saúde Sociedade 2007; 16(1): 18-27.

29. Rodrigues AAAO, Assis MMA. Oferta e demanda na atenção à saúde bucal: o processo de trabalho no Programa Saúde da Família em Alagoinhas - Bahia. Rev Baiana Saúde Pública 2005; 29(2):273-285.

30. O'Donnell O. Access to health care in developing countries: breaking down demand side barriers. Cad Saude Publica 2007; 23(12):2820-2834.

31. Castro MSM, Travassos C, Carvalho MS. Efeito da oferta de serviços de saúde no uso de internações hospitalares no Brasil. Rev Saude Publica 2005; 39(2): 277-284.

32. Oliveira EXG, Carvalho MS, Travassos C. Territórios do Sistema Único de Saúde: mapeamento das redes de atenção hospitalar. Cad Saude Publica 2004; 20(2):386-402.

33. Souza ECF. Acesso e acolhimento na atenção básica: uma análise da percepção dos usuários e profissionais de saúde. Cad Saude Publica 2008; 24(Supl.):s100-s110.

34. Marsiglia RMG, Silveira C, Carneiro Junior N. Políticas sociais: desigualdade, universalidade e focalização na saúde no Brasil. Saúde Sociedade 2005; 14(2):69-76.

35. Sousa MF. O Programa Saúde da Família no Brasil: análise do acesso à atenção básica. Rev Bras Enferm 2008; 61(2):153-158.

36. Costa NR, Pinto LF. Avaliação de programa de atenção à saúde: incentivo à oferta de atenção ambulatorial e a experiência da descentralização no Brasil. Cien Saude Colet 2002; 7(4):907-923.

37. Saito RXS, Lacerda RA, Fracolli LA. Atenção primária: análise de acesso ao primeiro contato em uma Unidade Básica de Saúde do Município de São Paulo. Rev Paul Enferm 2006; 25(2):74-81.

38. Solla J, Chioro A. Atenção ambulatorial especializada. In: Giovanella L, Escorel S, Lobato LVC, Noronha JC, Carvalho AI, organizadores. Políticas e sistema de saúde no Brasil. Rio de Janeiro: Fiocruz, Cebes; 2008. p. 627-665.

39. Dias-da-Costa JS. Utilização de serviços ambulatoriais de saúde em Pelotas, Rio Grande do Sul, Brasil: alguns fatores relacionados com as consultas médicas acima da média. Cad Saude Publica 2008; 24(2):353-363. 
40. Silva NN. Desigualdades sociais e uso de serviços de saúde: evidências de análise estratificada. Rev Saude Publica 2000; 34(1):44-49.

41. Feliciano KVO, Kovacs MH, Alzate A. Diagnóstico precoce da hanseníase: o caso dos serviços de saúde no Recife (Pernambuco), Brasil. Rev Panam Salud Publica 1998; 4(1):6-13.

42. Bahia L. As contradições entre o SUS universal e as transferências de recursos públicos para os planos e seguros privados de saúde. Cien Saude Colet 2008; 13(5):1385-1397.

43. Rodrigues MAP. Uso de serviços ambulatoriais por idosos nas regiões Sul e Nordeste do Brasil. Cad Saude Publica 2008; 24(10):2267-2278.

44. Oliveira EXG, Travassos C, Carvalho MS. Acesso à internação hospitalar nos municípios brasileiros em 2000: territórios do Sistema Único de Saúde. Cad Saude Publica 2004; 20(Supl. 2):S298-S309.

45. Castro MSM. Desigualdades sociais no uso de internações hospitalares no Brasil: o que mudou entre 1998 e 2003. Cien Saude Colet 2006; 11(4):987998.

46. Lima JC, Azoury EB, Bastos LHCV, Coutinho MM, Pereira NN, Ferreira SCC. Desigualdades no acesso e utilização de serviços de saúde no Brasil. Saúde Debate 2002; 26(60):62-70.

47. Neri M, Soares W. Desigualdade social e saúde no Brasil. Cad Saude Publica 2002; 18(Supl.):S77-S87.

48. Santos IS, Ugá MAD, Porto SM. O mix públicoprivado no Sistema de Saúde Brasileiro: financiamento, oferta e utilização de serviços de saúde. Cien Saude Colet 2008; 13(5):1431-1440.

49. Santos MAB, Gerschman S. As segmentações da oferta de serviços de saúde no Brasil: arranjos institucionais, credores, pagadores e provedores. Cien Saude Colet 2004; 9(3):795-806.

50. Acurcio FA, Guimaraes MDC. Uso de los servicios de salud y progresión al sida entre personas con infección por VIH en Belo Horizonte (Minas Gerais), Brasil. Rev Panam Salud Pública 1998; 4(5):331-340.

51. Sorj B. A democracia inesperada: cidadania, direitos humanos e desigualdade social. Rio de Janeiro: Jorge Zahar; 2004.

52. Araújo MAL, Leitão, GCM. Acesso à consulta a portadores de doenças sexualmente transmissíveis: experiências de homens em uma unidade de saúde de Fortaleza, Ceará, Brasil. Cad Saude Publica 2005; 21(2):396-403.

53. Braga PE, Cardoso MRA, Segurado AC. Diferenças de gênero ao acolhimento de pessoas vivendo com HIV em serviço universitário de referência de São Paulo, Brasil. Cad Saude Publica 2007; 23(11):2653-2662.

54. Lapa TM. Análise da demanda de casos de hanseníase aos serviços de saúde através do uso de técnicas de análise espacial. Cad Saude Publica 2006; 22(12):2575-2583.
55. Louvison MCP. Desigualdades no uso e acesso aos serviços de saúde entre idosos do município de São Paulo. Rev Saude Publica 2008; 42(4):733-740.

56. Moreira RS. A saúde bucal do idoso brasileiro: revisão sistemática sobre o quadro epidemiológico e acesso aos serviços de saúde bucal. Cad Saude Publica 2005; 21(6):1665-1675.

57. Almeida MF. Prevalência de doenças crônicas autoreferidas e utilização de serviços de saúde, PNAD/ 1998, Brasil. Cien Saude Colet 2002; 7(4):743-756.

58. Martins AMEBL, Barreto SM, Pordeus IA. Uso de serviços odontológicos entre idosos brasileiros. Rev Panam Salud Publica 2007; 22(5):308-316.

59. Travassos C, Viacava F. Acesso e uso de serviços de saúde em idosos residentes em áreas rurais, Brasil, 1998 e 2003. Cad Saude Publica 2007; 23(10):2490-2502.

60. Scatena LM, Villa TCS, Ruffino Neto A, Kritski AL, Figueiredo TMRM, Vendramini SHF, Assis MMA, Motta MCS. Dificuldades de acesso a serviços de saúde para diagnóstico de tuberculose em municípios do Brasil. Rev Saude Publica 2009; (3)43:389-397.

61. Diuana V. Saúde em prisões: representações e práticas dos agentes de segurança penitenciária no Rio de Janeiro, Brasil. Cad Saude Publica 2008; 24(8):18871896.

62. Pinheiro RS. Gênero, morbidade, acesso e utilização de serviços de saúde no Brasil. Cien Saude Colet 2002; 7(4):687-707.

63. Travassos C. Utilização dos serviços de saúde no Brasil: gênero, características familiares e condição social. Rev Panam Salud Publica 2002; 11(5-6):365-373.

64. Gomes R, Nascimento EF, Araújo FC. Por que os homens buscam menos os serviços de saúde do que as mulheres? As explicações de homens com baixa escolaridade e homens com ensino superior. Cad Saude Publica 2007; 23(3):565-574.

65. Pagliuca LMF, Aragão AEA, Almeida PC. Acessibilidade e deficiência física: identificação de barreiras arquitetônicas em áreas internas de hospitais de Sobral, Ceará. Rev Esc Enferm USP 2007; 41(4):581-588.

66. Carneiro FF. Saúde de famílias do Movimento dos Trabalhadores Sem Terra e de bóias-frias, Brasil, 2005. Rev Saude Publica 2008; 42(4):757-763.

67. Santos AM, Assis MMA. Da fragmentação à integralidade: construindo e (des) construindo a saúde bucal no Programa de Saúde da Família (PSF) de Alagoinhas, BA. Cien Saude Colet 2006; 11(1):53-61.

68. Santos AM, Assis MMA, Nascimento MAA, Jorge MSB. Vínculo e autonomia na prática de saúde bucal no programa saúde da família. Rev Saude Publica 2008; 42(3):53-61.

69. Castoriadis C. O mundo fragmentado. Encruzilhadas do Labirinto III. Rio de Janeiro: Paz e Terra; 1992. 